\title{
The Preparation and Processing Technology of PLA Superfine Fiber Non-woven Materials
}

\author{
He QIU ${ }^{1, a}$, Wei ZHANG ${ }^{1, b}$, Qi-Wen LU ${ }^{1, c}$, Ting-Ting HUANG ${ }^{1, d}$, Xin XU ${ }^{1, e}$, \\ Yu ZHANG ${ }^{1, f, *}$ \\ ${ }^{1}$ College of Textiles, Nantong University Nantong, Jiangsu 226019, China

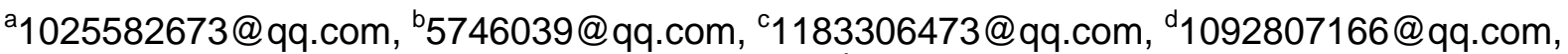 \\ e1220890645@qq.com, 'rz.yu@ntu.edu.cn \\ ${ }^{*}$ Corresponding author
}

Keywords: PLA, Superfine Fiber, The Preparation Process, Performance.

\begin{abstract}
In view of the disadvantages of superfine fiber nonwovens materials made by polypropylene (PP), such as poor hydrophilcity, deficiency of antibacterial property, poor hand feeling, we chose polylactic acid (PLA) slices as the raw material to make superfine fiber nonwoven materials, which is quite antibacterial and very soft. Using a modified superfine fiber processing technology, the preparation of PLA superfine fiber non-woven materials could be easy and effective. The fiber fineness of PLA materials is mainly distributed in 1-5 microns, and the contact Angle of PLA materials is about 50 degrees lesser than that of PP materials. The antibacterial properties of PLA are good enough. We discussed the problems in the process of production and gave some suggestions.
\end{abstract}

\section{Introduction}

In recent years, influenced by the A (H1N1) influenza and haze weather, the melt-blown superfine fiber non-woven materials gradually become the focus concerned by scholars and manufacturers. The unique superfine fiber structure of melt-blown non-woven materials can provide it with high hydrostatic pressure ${ }^{[1]}$ which make the materials with good barrier property and filtering performance. Besides, with the excellent permeability and light weight, the melt-blown superfine fiber non-woven materials are widely used in medical and health field. ${ }^{[2]}$ The warm effect of the materials is also excellent. But as people's living standards continue to improve, the market increasingly need functional products. However, traditional superfine fiber non-woven materials made by PP feel stiff, and the hydrophilcity and antibacterial property are very poor, which influence the application of such products seriously. ${ }^{[3]}$ Besides, traditional raw material depends on the non-renewable oil resources, and the material waste is non-biodegradable. All these factors are not conducive to the sustainable development of the social resources.

On the contrary, ultrafine fiber non-woven materials made by PLA chips could be a very good compensation for all the weaknesses of PP materials. The crystallinity of PLA polymer is very low, and materials production made by it feels soft. Polymer molecules contain a certain amount of hydroxy hydrophilic group, which make the material with certain hydrophilic. In addition, the PLA itself has good antibacterial property and function of suppressing mite. Polylactic acid is mainly derived from plants and material waste is biodegradable under natural condition. Thus it is a kind of environmentally friendly material. ${ }^{[4]}$

\section{Preparation Technology}

\section{Raw Materials and Equipments}

Materials: PLA polymer slices (made by Nature Works company, America), the properties are shown in Table1. 
Tab.1 The Performance Parameter of PLA Material

\begin{tabular}{ccc}
\hline $\begin{array}{c}\text { Performance } \\
\text { indicators }\end{array}$ & Test standard & Test data \\
\hline Density $\left(\mathrm{g} / \mathrm{cm}^{3}\right)$ & ASTM D792 & 1.24 \\
$\begin{array}{c}\text { melt index } \\
(\mathrm{g} / 10 \mathrm{~min})\end{array}$ & ASTM D1238 & 70 to 85 \\
$\mathrm{~T}_{\mathrm{g}}\left({ }^{\circ} \mathrm{C}\right)$ & ASTM D3417 & 55.0 to 60.0 \\
$\mathrm{~T}_{\mathrm{m}}\left({ }^{\circ} \mathrm{C}\right)$ & ASTM D3418 & 160 to 170
\end{tabular}

Processing equipments: ROC-MB300 melt-blown non-woven experimental equipment (researched by Institute of textile and apparel of Nantong University together with Nantong liyang new material development co., LTD); 101AB-1 thermostatic drum wind drying oven (provided by HengChang instrument factory, Shanghai hualian environmental testing equipment company).

\section{The Production Process}

The technological process of PLA superfine fiber non-woven materials is as follows: Drying of polymer chips $\rightarrow$ feeding of polymer chips $\rightarrow$ melt extrusion $\rightarrow$ fiber forming and cooling $\rightarrow$ web formation $\rightarrow$ fiber bonding (can be self bonding or hot glue) $\rightarrow$ trimming winding $\rightarrow$ after finishing or special finishing (heat styling, antibacterial, water softening and etc.).

\section{The Processing Technology}

To prevent the hydrolysis of PLA in spinning process, strict drying of polymer chips is need before processing. The drying process parameters are as follows: drying under $70^{\circ} \mathrm{C}$ for 24 hours. The rest heating section temperatures of the spinning are shown in Table2.

As the viscosity of polymer is high, the melt index of PLA slice is relatively low. Thus the speed of the extruder should not be too fast. The die swell ratio of PLA is bigger than that of PP, so the speed of the metering pump should be reduced appropriately to control the fineness of the fiber.

Tab.2 Heating Section Temperatures of the Equipment

\begin{tabular}{cc}
\hline Section & Temperature \\
\hline Extruder & $180^{\circ} \mathrm{C}$ \\
Filter screen & $185^{\circ} \mathrm{C}$ \\
Metering pump & $190^{\circ} \mathrm{C}$ \\
Material road & $190^{\circ} \mathrm{C}$ \\
Die head & $210^{\circ} \mathrm{C}$ \\
Hot air & $240^{\circ} \mathrm{C}$ \\
\hline
\end{tabular}

\section{Material Performance Test and Analysis \\ TG and DSC Test}

The thermodynamic performance test of PLA superfine fiber materials is conducted, and the data is shown in Figure1. 

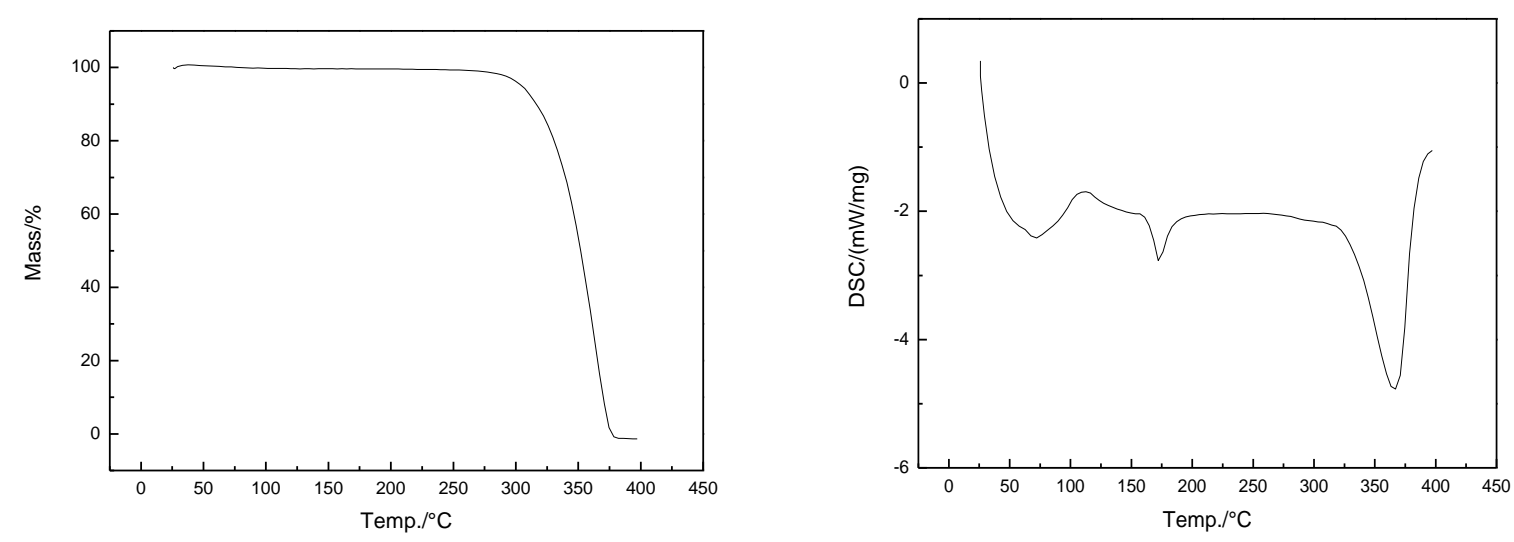

Fig.1 The TG and DSC Curves of PLA Microfiber Material

As is shown in the TG curve, when the temperature is less than 300 degrees Celsius, the thermodynamic properties of the material are very stable. Once the temperature reaches the decomposition point of the material, it decomposes rapidly.

It can be seen in the DSC curve that the glass transition temperature of the material is between 60 and 70 degrees Celsius. The endothermic peak in the melting point $\left(170^{\circ} \mathrm{C}\right)$ of the material is not particularly outstanding, illustrating that the crystallinity of the material is not very high and the proportion of the amorphous area is relative large, thus making the material with soft handle. The decomposition point of the material is around $350^{\circ} \mathrm{C}$, so the material should be processed and used below the temperature as far as possible. There are two obvious endothermic peaks around $70^{\circ} \mathrm{C}$ and $170^{\circ} \mathrm{C}$, indicating that the process parameters of the heating sections afterwards should be within this temperature range.

\section{SEM Test}

The samples are observed under scanning electron microscope after spraying gold.

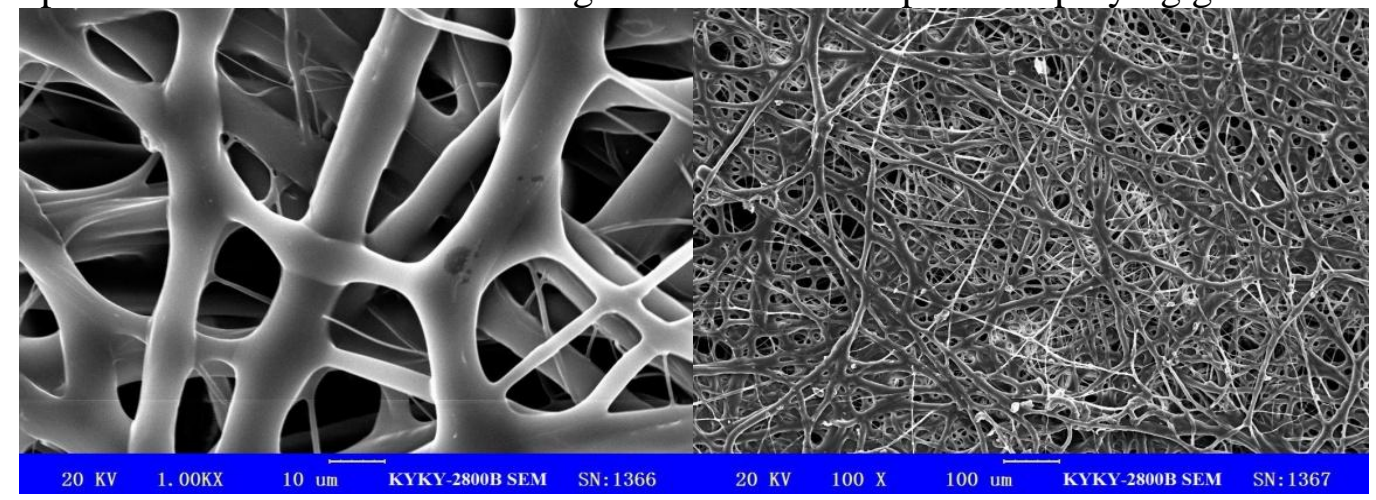

Fig.2 The SEM Observation Images of PLA Superfine Fiber Material

The fiber diameters are mainly distributed in 0.5-10 microns, among which 1-5 microns are most common. With the ultra-fine fiber structure, materials have a good resistance of bacteria and excellent filtration performance. The warm effect of the materials is also excellent.

\section{Antibacterial Performance Test}

Testing method: coating the Colombia petri dishes with a layer of E. coli liquid(the concentration is $0.5 \%$ ), cutting the samples into circles with $20 \mathrm{~mm}$ diameter and put them on the petri dishes smoothly. Cultivating under the constant temperature of $36^{\circ} \mathrm{C}$ for 24 hours. Testing results are shown in Figure3. 


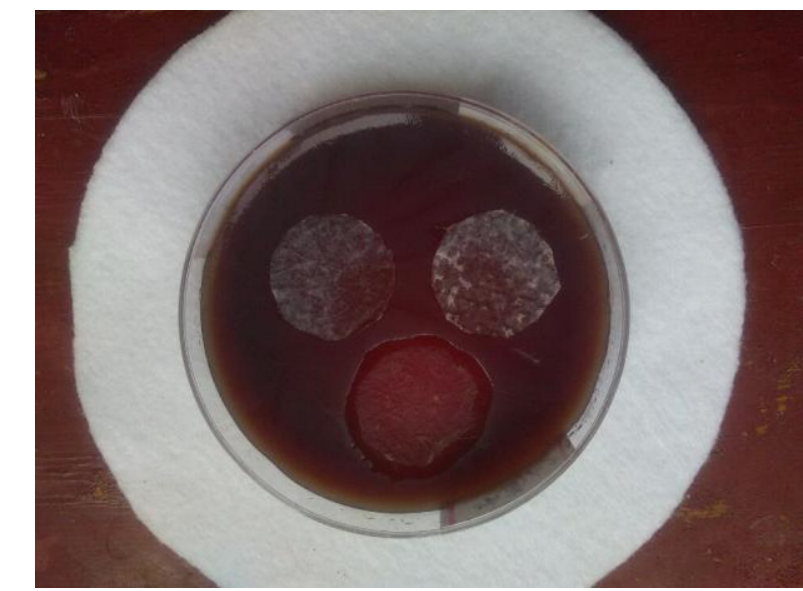

(Above for PP Materials, Below for PLA Materials)

Fig.3 The Antibacterial Test Results of PLA Materials and PP Materials

As is shown in Figure 3, there are obvious bacteriostatic circles around PLA superfine fiber materials. The antibacterial property of PLA materials is so good that they are very suitable for applying in the field of medical, health care and clothing.

\section{Contact Angle Test}

Using distilled water to test the contact Angle of PP material and PLA material respectively, and the goniometric method was used to measure the contact angle, the testing results are shown in Figure4.

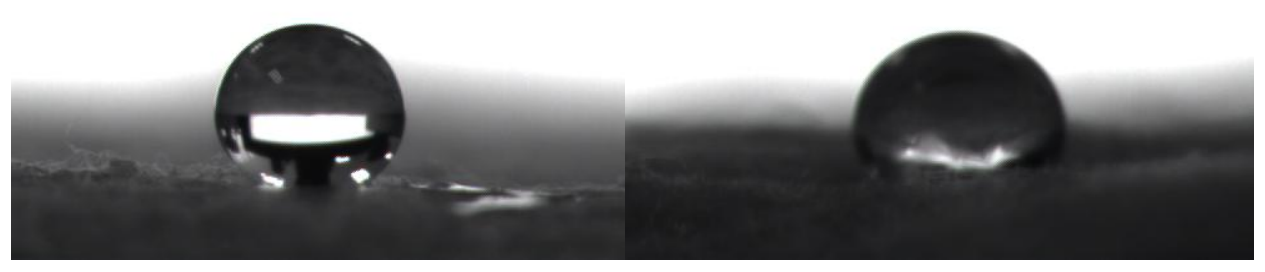

Fig.4 Contact Angle Measurement of PP Materials and PLA Materials

The testing results shows that the contact Angle of PP material with water is $158^{\circ}$, whereas the contact Angle of PP material is only $109^{\circ}$, indicating that the hydrophilicity of the PLA material is much better than traditional materials made by pp.

\section{Summary}

(1) The raw material of PLA nonwovens can be either derived from oil or renewable crops (corn, wheat, etc.), thus it is a kind of renewable material. In addition, PLA polymer is completely biodegradable, development of the material could reduce the environment pollution caused by petroleum chemical industry and material wastes. However, limited by its own properties, PLA non-woven materials cannot be applied in mass industrial production immediately. ${ }^{[5]}$ With the improvement of the melt-blown technology and synthetic technology, it is convincing that the PLA melt-blown nonwovens could be used more and more widely in the future. ${ }^{[6]}$

(2) Though it is a kind of biodegradable material, PLA nonwovens are very stable when used at room temperature and they are also good biocompatible materials. The fiber diameters of the materials are mainly distributed in 1-10 microns, wherein 2-5 microns are most common. With 
superfine fiber structure, the PLA materials have good antibacterial properties and filtration performance.

(3) Polymer chips with high melt index are often used for melt-blown processing, the viscosity is low, thus the reynolds number in the non-Newtonian fluid tube is large. Inertia force dominates; polymer is not easy to be delayed on the pipe wall. However, the melt index of PLA polymer is low and the viscosity is high, thus the reynolds number in fluid tube is small. Adhesive force affects significantly, polymer may be delayed on the pipe wall and eventually carbonizes. With the time increasing, the PLA polymer delayed on the pipe wall accumulates, that may cause the uneven flow of the liquid and even clog the pipes or the die head. So the industrialized production of the PLA melt-blown nonwovens should adopt smooth pipe wall with low resistance to the polymer. And the melt flow properties of PLA polymer should be improved as much as possible.

(4) Compared with PP, the non-newtonian index of PLA polymer is closer to 1, so the stress relief when the melt rushes out of the jet hole is more obvious. Die swell ratio increases significantly. Suggestions are that the speed of the hot air should be increased or spinneret components with smaller bore diameter should be applied to ensure superfine fiber structure. Besides the decomposition point of PLA is low, so the processing temperature could not be too high. To ensure the sufficient bonding of the fibers, the receiving distance should be low or the hot rollers should be used to receive the materials. Tubes should be cleaned timely after the melt-blown processing of PLA polymers, removing residual materials and avoiding adverse effects to next processing.

\section{Acknowledgement}

This work is supported by Nantong University graduate student research and innovation program (No. YKC15023). This work was also supported by National Natural Science Foundation of China (No.51303085) and National College Students Innovation and entrepreneurship training program in Nantong University (No. 201510304030).

\section{References}

[1] THOMAS S, MCCUBBIN P. An in Vitro Analysis of the Antimicrobial Properties of 10 Silver-containing Dres-sings [J]. J Wound Care, 2003, 12(8):305-308.

[2] GUEST J F,RUIZ F J,MIHAI Aet al. Cost Effectiveness of Using Carboxymethylcellulose Dressing Compared with Gauze in the Management of Exuding Venous Leg Ulcers in Germany and the USA [J]. Curr Med Res Opin, 2005, 21(1):81-92.

[3] LAY-FLURRIE K. The Properties of Hydrogel Dressin-gs and Their Impact on Wound Healing [J]. Prof Nurse, 2004, 19(5):269-273.

[4] YAMANAKA.Lactrona biodegradable fiber development and applications [J]. Chemical Fibers International, 1999, 49(12):501-503.

[5] BO Shi, DORIS P. Enhanced mineralization of PLA melt blown materials due to plasticization [J]. J Polym Environ, 2010(18):122-127.

[6] LI Yongjin, HIROSHI S. Toughening of polylactide by melt blending with a biodegradable poly (ether) urethane elastomer [J].Macromolecular Bioscience, 2007, 7(7):921-928. 